\title{
Scapular Rotation in Swimmers with and without Impingement Syndrome: Practice Effects
}

\author{
KA PIK EVA SU ${ }^{1}$, MICHAEL P. JOHNSON ${ }^{3}$, ED J. GRACELY ${ }^{2}$, and ANDREW R. KARDUNA ${ }^{4}$ \\ ${ }^{1}$ Programs in Rehabilitation Sciences, ${ }^{2}$ Family, Community, \& Preventive Medicine, Drexel University, Philadelphia, PA; \\ ${ }^{3}$ Department of Physical Therapy, University of the Sciences in Philadelphia, Philadelphia, PA; and ${ }^{4}$ Department of \\ Human Physiology, University of Oregon, Eugene, OR
}

\begin{abstract}
SU, K. P. E., M. P. JOHNSON, E. J. GRACELY, and A. R. KARDUNA. Scapular Rotation in Swimmers with and without Impingement Syndrome: Practice Effects. Med. Sci. Sports Exerc., Vol. 36, No. 7, pp. 1117-1123, 2004. Purpose: The purpose of this study was to examine the effects of a normal swim practice on the scapular kinematics of swimmers with impingement syndrome and healthy swimmers. Methods: Twenty swimmers with no known shoulder pathology and 20 swimmers with shoulder impingement syndrome participated in this study. Shoulder strength measurements were made with a hand-held dynamometer. Static scapular upward rotation was measured with an inclinometer with the arm at rest, and at 45, 90, and $135^{\circ}$ of humeral elevation. Measurements were made pre- and postswim training. Results: There were no differences in baseline measurements of kinematics between the two groups. After swimming, both groups experienced muscle fatigue as indicated by a significant reduction in force generation. Although swimming practice resulted in no significant differences in scapular kinematics for the healthy swimmers, there were significant decreases in scapular upward rotation in subjects with shoulder impingement. Conclusions: Abnormal scapular kinematics in swimmers with impingement syndrome may only be observed after an intense swim practice. The examination of swimmers immediately after swimming may provide more information regarding impingement syndrome than a typical clinical exam. Key Words: BIOMECHANICS, KINEMATICS, SWIMMING, SHOULDER, FATIGUE
\end{abstract}

$\mathrm{S}$ wimming ranks above both hiking and jogging in the number of participants in the United States (16). Competitive swimming is a demanding and timeconsuming sport, with athletes at the elite level practicing as many as $20-30 \mathrm{~h} \cdot \mathrm{wk}^{-1}$. During $1 \mathrm{yr}$ of practice, the average top-level swimmer may perform over 500,000 strokes per arm (30). It is perhaps not surprising that the prevalence of shoulder pain in competitive swimmers ranges from 10 to $60 \%$ and tends to increase the longer the athlete participates in the sport $(4,21)$.

Not unlike shoulder disorders in the workplace, overuse is believed to be one of the chief factors responsible for shoulder pain in swimmers $(13,16)$. Most successful competitive swimmers in the United States practice several hours a day, $5-7 \mathrm{~d} \cdot \mathrm{wk}^{-1}$ for 10 or 11 months each year (13). Innumerable repetitions over many years of hard training, together with an increased muscular imbalance around the shoulder girdle, seem to be the main etiological factors in

\footnotetext{
Address for correspondence: Andrew Karduna, Ph.D., University of Oregon, Department of Human Physiology, Eugene, OR 97403; E-mail: karduna@uoregon.edu.

Submitted for publication May 2003.

Accepted for publication February 2004.
}

0195-9131/04/3607-1117

MEDICINE \& SCIENCE IN SPORTS \& EXERCISE E $_{\circledast}$

Copyright (C) 2004 by the American College of Sports Medicine

DOI: 10.1249/01.MSS.0000131955.55786.1A the development of overuse syndrome; often referred to as swimmer's shoulder $(13,27,32)$. More specifically, impingement syndrome $(1,31)$, as originally described by Neer (22), is common among swimmers.

In the general population, a link has recently been established between impingement syndrome and alterations in scapular kinematics $(7,17,18)$. This is of concern to the competitive swimmer because of the crucial role the scapula plays in providing a stable base for the glenohumeral joint during swimming (28). Several studies have demonstrated altered EMG activity of the scapulothoracic muscles in swimmers with shoulder pain $(26,27,32)$. However, to our knowledge, the only studies of scapular kinematics in swimmers have been performed in individuals without any type of shoulder pathology $(5,36)$.

Consequently, the goal of this research study was to investigate scapular kinematics in swimmers with no known shoulder pathology and in swimmers with shoulder impingement syndrome. Our hypothesis was that changes in scapular kinematics due to swim practice would be greater in subjects with shoulder impingement syndrome when compared with healthy swimmers.

\section{MATERIALS AND METHODS}

\section{Subjects}

Approximately 50 swimmers from the swim teams of local universities and swim clubs were screened at poolside, 
and 40 swimmers who fit the inclusion/exclusion criteria participated. Subjects included 20 healthy swimmers with no known shoulder pathology and 20 swimmers with shoulder impingement. The healthy swimmers were recruited to match the swimmers with impingement in terms of their dominant swim style. Subjects with impingement were tested on their pathologic side, and healthy subjects were tested on the same side as the impingement swimmer to whom they were matched.

The inclusion criteria for all subjects were: 1) age between 18 and $35 \mathrm{yr}, 2)$ competitive swimming experience of more than $5 \mathrm{yr}$, and 3) current swimming training schedule for more than $2 \mathrm{~d}$ and $10 \mathrm{~km} \cdot \mathrm{wk}^{-1}$. The exclusion criteria for all subjects were: 1) a history of cervical or thoracic pathology; 2) less than $135^{\circ}$ of active humeral elevation in the scapular plane; 3 ) previous shoulder surgery; 4) previous shoulder injury in the past six months; 5) presence of shoulder instability based on a positive sulcus test, anterior, or posterior apprehension tests; and 6) presence of shoulder pain that prevented the correct execution of the tests, including inability to achieve relaxation during testing.

There were additional inclusion criteria for each group. For the subjects with impingement, they were: 1) symptoms of shoulder pain which interfered with the swim training and had lasted for at least 1-wk duration, and 2) the shoulder pain had to be at phase II or phase III according to the Neer and Welsh swimmer's shoulder grading system (22). Essentially, this meant either nondisabling pain during and after workouts, or disabling pain during and after workouts that interferes with the swimmer's performance; 3 ) at least three of the following six criteria indicating impingement at the shoulder: a) positive Neer test, b) positive Hawkins test, c) pain with active shoulder elevation in the scapular plane, $d$ ) pain with palpation of the rotator cuff tendons, e) a history of pain in the C5-C6 dermatome, and f) pain with resisted isometric abduction. Additional inclusion criteria for the healthy swimmers were: 1) no shoulder pain in the past 6 months, and 2) negative response to all six impingement signs listed above.

All of the measurements and screening tests were performed by a physical therapist with $8 \mathrm{yr}$ of orthopedic clinical experience. Before participation, all subjects were informed of the nature of the study and then read and signed the consent form approved by the university Internal Review Board. The subjects also had to complete a questionnaire, which provided us with information regarding demographics, dominant swim style, and issues related to the inclusion/exclusion criteria listed previously. All swimmers were symptom free before swimming.

\section{Instrumentation}

Kinematics. A Pro-360 inclinometer (Pro-360) (Macklanburg Duncan, Oklahoma City, OK) was used to assess static positions of scapular upward rotation. Two wooden "Y" shaped locator rods were attached to the bottom of the instrument (Fig. 1A). These locator rods were placed over the scapular spine: one on the posterolateral aspect of the
A

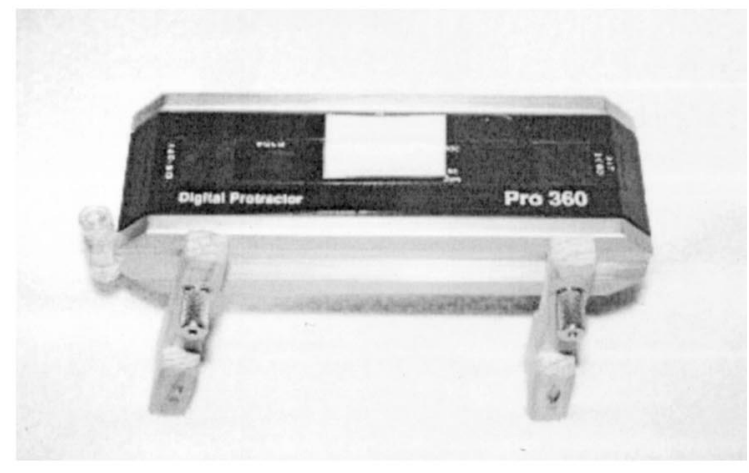

\section{B}

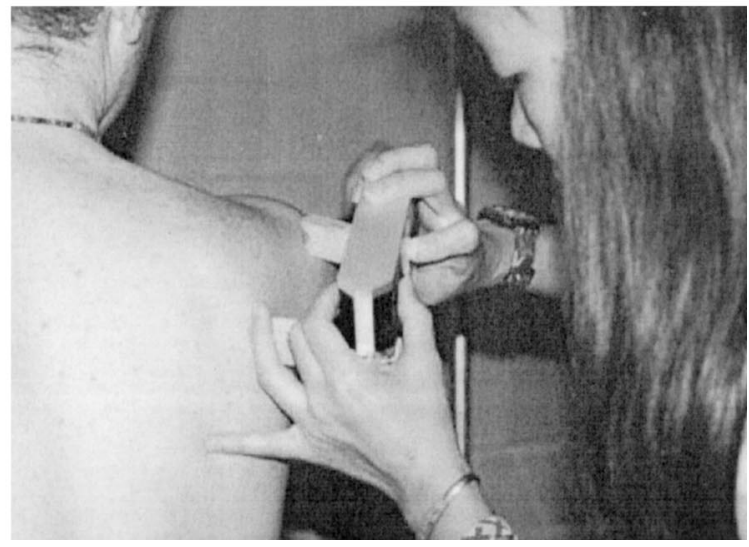

FIGURE 1-A. Modified digital inclinometer. B. Application of the inclinometer to the scapular of a subject, at $90^{\circ}$ of elevation.

acromion laterally and one on the root of the scapular spine medially (Fig. 1B). A small bubble level was attached along the side of the Pro-360 to allow for consistent positioning of the device in reference to the plane in which upward rotation occurred. The window display of the Pro-360 was covered in order to blind the tester from the readout and prevent bias during measurements. We have previously demonstrated that this device is valid and reliable for assessing upward rotation of the scapula about an axis perpendicular to the plane of the scapula (14).

Subjects stood facing a 3-m tall guiding pole that was placed $1 \mathrm{~m}$ in front of them in the scapular plane $\left(40^{\circ}\right.$ anterior to the coronal plane). There were gradation marks along the pole to provide subjects with a reference for sustained humeral elevation. During data collection, subjects were asked to stand in an erect position to minimize excessive postural sway, as well as to maintain the arm with elbow and finger extension and forearm supination.

The investigator placed the inclinometer on the arm just proximal to the subjects' elbow joint. The subjects were then requested to elevate their arm along the plane of the guiding pole and asked to stop when their arm reached the desired humeral elevation. While they maintained this elevation, the inclinometer was removed from the subjects' arm and placed on the spine of the scapula, as described above. Once the inclinometer was in position, its numeric 


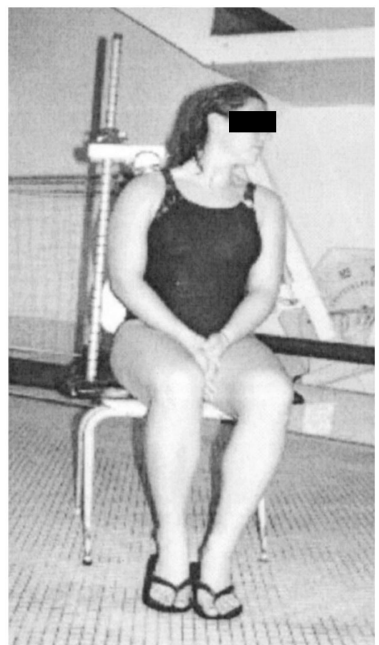

B

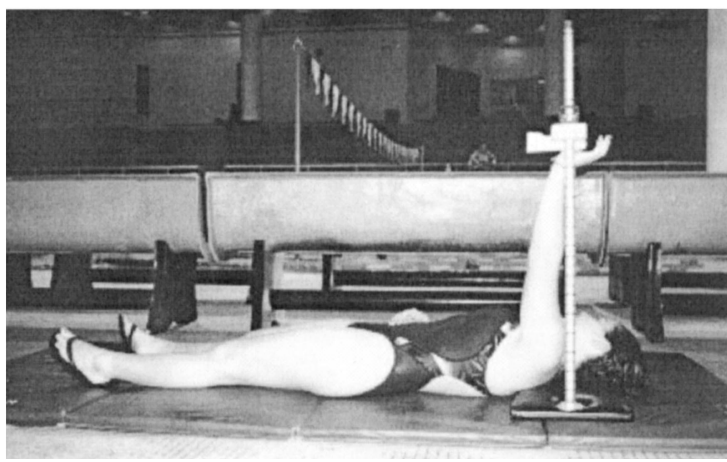

FIGURE 2-Positioning of subject for strength testing. A. Shrug test: designed to assess the strength of the upper trapezius. B. Punch test: designed to assess the strength of the serratus anterior.

value on the readout was stored in memory, and the subject lowered his or her arm. The investigator then retrieved the data point. The subjects' scapulae were measured at four positions: 0 (rest), 45,90 , and $135^{\circ}$ of humeral elevation.

Force. A Nicholas Model 01160 Hand Held Dynamometer (HHD) (Lafayette Instrument, Lafayette, IN) was used to assess muscle strength, which was defined as the maximum isometric voluntary force that subjects were able to exert under specific testing conditions. The HHD was attached to a wooden stabilizer that was made to conform to the curvature of the device. The HHD could rotate $15^{\circ}$ within the wooden stabilizer to permit it to be adjusted to fit different slopes of the subjects' shoulders. The whole unit was then connected to a padded wooden base through a metal pole. The wooden stabilizer and the HHD were able to slide along the metal pole. The whole unit was designed so that the subjects' weight on the wooden base would stabilize the system.

To assess the strength of the upper trapezius muscle, a shrug maneuver was performed. Subjects sat on the wooden base on top of a chair (Fig. 2A). The height and the rotation angle of the HHD were adjusted so as to place it on the acromion process of the tested arm. Before testing, subjects were instructed to turn their heads toward the contralateral side. Subjects practiced three repetitions of submaximal shoulder shrug and then performed a maximum isometric shrug for $5 \mathrm{~s}$. The subjects rested for 1 min after which the test was repeated.

To assess the strength of the serratus anterior, a punch maneuver was performed. The subjects lay supine on a pad that was placed on top of the wooden base (Fig. 2B). The tested arm was maintained with elbow and wrist extension and forearm pronation. The arm was flexed to $90^{\circ}$ in the sagittal plane. The height of the HHD was adjusted so that the heel of the palm was against the HHD. As with the shrug, subjects practiced three repetitions of submaximal shoulder shrug and then performed a maximum isometric shrug for $5 \mathrm{~s}$ and then repeated this after a 1-min rest period.

Protocol. All testing was performed at the pool area where subjects normally practiced. Two sets of measurements were made, one before getting in the pool and one immediately after the completion of a swim practice of their usual routine under the guidance of their coach. The distance that the subjects practiced in each swim style (e.g., freestyle, butterfly), and the swim practice duration were recorded. All measurements were made with subjects wearing a bathing suit so that the scapulae were exposed.

Subjects were tested for strength (shrug and punch) and kinematics (rest, 45, 90, and $135^{\circ}$ of humeral elevation). Each strength and kinematic measurement was performed twice. The order of testing (shrug, punch, and kinematics) was randomized to prevent a sequencing effect. Furthermore, the order of humeral elevations was also randomized.

\section{Data Analysis}

Intrarater test-retest reliability between the two trials for shoulder shrug and punch strength tests, as well as static scapular upward rotation were summarized by the intraclass correlation coefficients, ICC $(3,1)$, and the standard error of the measurement (SEM). The mean of the two trials was used for subsequent data analysis. In order to assess strength, a two-way, repeated measures ANOVA was performed on the force data, with one within subject factor, practice (pre and post), and one between subject factor, group (healthy vs impingement). This two-way ANOVA returns an effect for the two main effects and an effect for interaction between them. The interaction tests whether there is more change over time between one group than the other and is thus the most direct test of our hypothesis. To assess changes in kinematics, a three-way, repeated measures ANOVA was performed, with two within-subject factors, practice (pre and post) and position (four humeral positions), and one between-subject factor, group (healthy vs impingement). The dependent variable for the three-way ANOVA was scapular orientation (as measured by the inclinometer). Based on the results of the three-way ANOVA, follow-up two-way ANOVAs were performed. For the purposes of this study, significance was set at the 0.05 level. Statistics were run using SPSS, version 11.0 (SPSS Inc., Chicago, IL). 
TABLE 1. Comparison of demographic data between the two groups of swimmers.

\begin{tabular}{lccc}
\hline & $\begin{array}{c}\text { Swimmers } \\
\text { Swimmers } \\
\text { Swith }\end{array}$ & $\begin{array}{c}\text { Swithy } \\
\text { Impingement }\end{array}$ & $\boldsymbol{P}$ \\
\hline Gender & $10 \mathrm{~F}, 10 \mathrm{M}$ & $11 \mathrm{~F}, 9 \mathrm{M}$ & 0.66 \\
Age $(\mathrm{yr})$ & $23.6 \pm 4.6$ & $24.2 \pm 6.5$ & 0.76 \\
Height $(\mathrm{cm})$ & $178 \pm 10$ & $174 \pm 8$ & 0.28 \\
Weight $(\mathrm{kg})$ & $72 \pm 10$ & $70 \pm 12$ & 0.72 \\
Competitive experience $(\mathrm{yr})$ & $12.9 \pm 4.5$ & $14.2 \pm 5.0$ & 0.39 \\
Practice duration $\left(\mathrm{h} \cdot \mathrm{wk}^{-1}\right)$ & $7.6 \pm 5.3$ & $8.1 \pm 5.1$ & 0.80 \\
Practice distance $(\mathrm{km})$ & $3.3 \pm 1.1$ & $3.0 \pm 0.9$ & 0.33 \\
\hline
\end{tabular}

\section{RESULTS}

The swim practice session ranged from 1 to $2 \mathrm{~h}$ for all participants. The number of subjects that selected each swim style was identical for both groups: freestyle $(N=8)$, breast $(N=6)$, butterfly $(N=4)$, and back $(N=2)$. Besides the four basic swim styles, they also practiced kicking with a kick board and isolated arm motions by using a float bell between their legs. The total swim distance for each swimmer ranged from 1.7 to $6.7 \mathrm{~km}$. There were no significant differences in gender, age, height, weight, competitive experience, practice duration, and practice distance between the two groups (Table 1). The ICC $(3,1)$ values ranged from 0.97 to 0.99 for the forces measurements and from 0.87 to 0.99 for the kinematic measurements.

The results of the two-way ANOVAs for strength revealed that there was a significant reduction in force after the swim practice for both the shrug and punch $(P<0.001)$. However, there were no significant interactions between practice and group, indicating that both groups had similar drops in strength. When the data from both groups were combined, there was a $14 \%$ reduction in shrug strength and a $13 \%$ reduction in punch strength (Fig. 3).

The results of the three-way ANOVA indicated a significant effect of practice $(P<0.001)$, elevation angle $(P<$ $0.001)$, and group $(P=0.044)$. Additionally, there was a significant interaction between the two effects of interest:

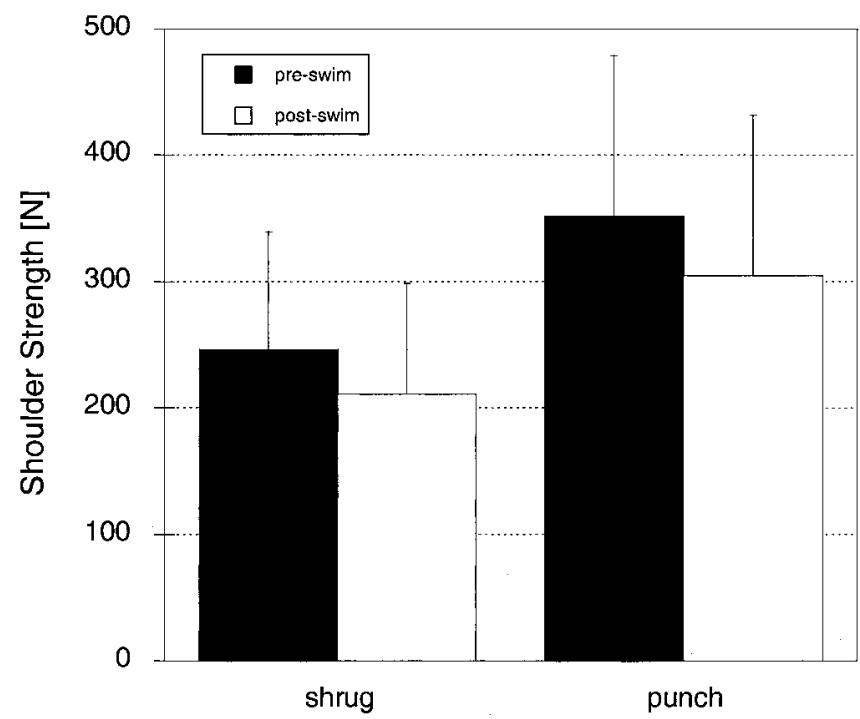

FIGURE 3-Mean and SD of force production for both groups of subjects, before and after swimming practice. practice and group $(P=0.003)$. Consequently, to further investigate these differences, the data were reanalyzed using two-way ANOVAs.

With practice taken out of the analysis, two follow-up two-way ANOVAs were run, one before the swim practice and one after. Although the two groups were not significantly different before the swim practice $(P=0.2)$, there was a significant difference after the swim practice $(P<$ $0.0082)$. Analysis of the results indicates that this effect was more pronounced at higher elevation angles (Fig. 4).

When group was taken out of the analysis, two additional follow-up two-way ANOVAs were run, one for the subjects with impingement and one for the healthy swimmers. Although there was no effect of the swim practice on the healthy subjects $(P=0.21)$, there was a significant effect on the swimmers with impingement $(P<0.0001)$.

\section{DISCUSSION}

Reliability for the present study was found to be good (29) for the measurement of scapular upward rotation at all humeral elevation positions, although ICC values were higher in the dependent position and tended to decrease as humeral elevation increased in both groups. One possible explanation for this may be more effective palpation of bony landmarks at lower elevations due to less muscular contraction. Additionally, it is inherently more difficult to maintain and repeat a specific humeral position at higher elevation angles, due to the higher forces required. The shrug and punch tests also demonstrated excellent reliability. A contributing factor to this was that when the subjects sat and laid down on the wooden base of the device, they were able to use their own body weight to stabilize the device from excessive motion during the shoulder shrug and punch.

Although there are many muscles that assist with scapular motion, the three muscles that are traditionally attributed as upward rotators are the upper trapezius, lower trapezius, and serratus anterior $(24,25)$. The lower trapezius was not evaluated in this study because of the difficulty in performing an isolated muscle test. As expected, due to the intense swim training, significant drops in force production were noted. One possible explanation for this reduction is that the serratus anterior and upper trapezius muscles were fatigued as a result of the swim practice. Because the performance of the swimmers was not the goal in this study, the lap time of the swimmers was not recorded. It is therefore not possible to comment on the relationship between fatigue and swimmers' performance. Positions of scapular upward rotation in the present study were higher when compared with other two-dimensional studies involving measurements of this variable. However, previous studies did not focus on an athletic population $(6,14,37)$. The subjects who participated in the present study were all competitive swimmers, practicing between 3 and $18 \mathrm{~h} \cdot \mathrm{wk}^{-1}$. Some of their upper extremities performed repeated overhead motions approximately $7000 \times \mathrm{wk}^{-1}$. The noted increase in mobility of the swimmer's scapulothoracic joint might be a necessary adaptive change to this repetitive loading. 
$0^{\circ}$ of elevation

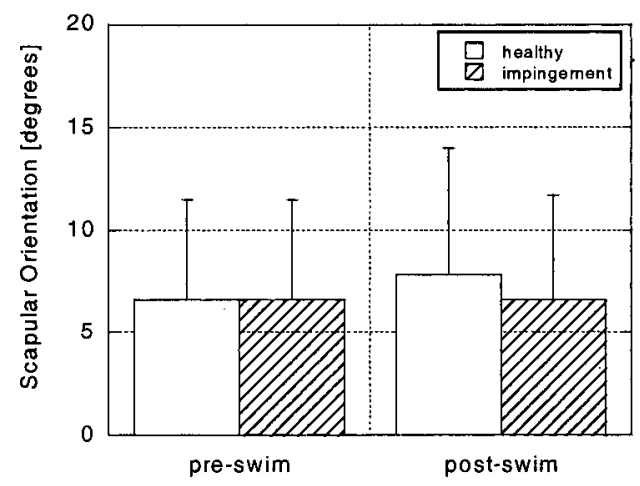

$90^{\circ}$ of elevation

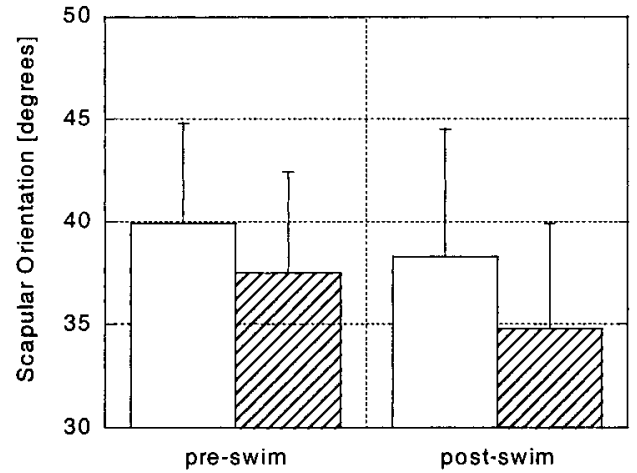

$45^{\circ}$ of elevation

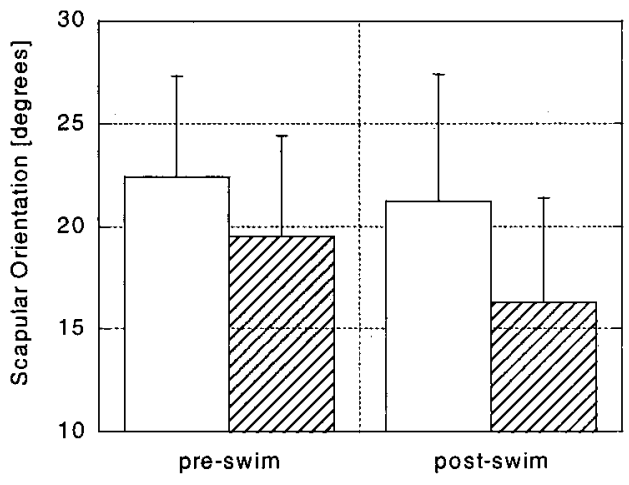

$135^{\circ}$ of elevation

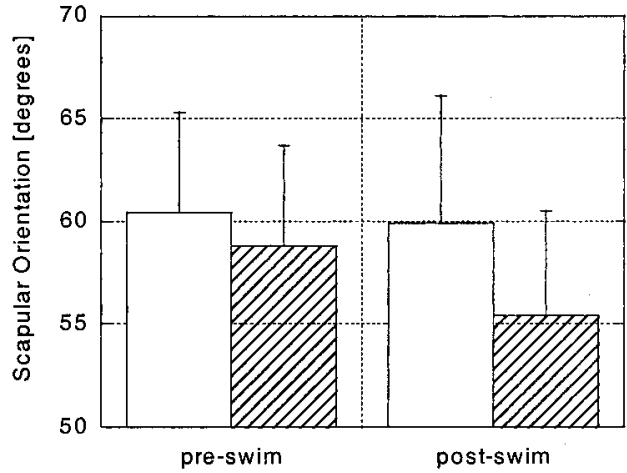

FIGURE 4-Scapular orientation for all humeral elevation angles: 0 (rest), 45,90 , and $135^{\circ}$. Graphs represent the means and SD for both groups of subjects, before and after swimming practice.

Our results showed that the two groups had similar scapular upward rotation patterns before swim practice. A previous two-dimensional study of postural differences at rest between healthy subjects and subjects with shoulder overuse injuries found no significant differences in scapular upward rotation (11). An examination of previous 3D studies comparing healthy subjects with patients with impingement syndrome reveals conflicting results, with some studies demonstrating a decrease in upward rotation with impingement $(7,17)$ and some studies finding no significant differences $(10,12,18)$.

Our results showed that the two groups demonstrated similar scapular upward rotation patterns before swim practice. However, after practice the impingement group demonstrated less upward rotation, particularly at 45, 90, and $135^{\circ}$ of humeral elevation. These results are perhaps not surprising, given our subject population. Based on our inclusion criteria, all subjects with impingement reported pain during and after the workout, but subjects who reported shoulder pain before practice were excluded. So essentially, differences were only noted when the subjects with impingement experienced pain. Future studies involving swimmers who present with shoulder pain before the workout are recommended.

With the use of magnetic resonance imaging in an in vivo setting, Solem-Bertoft et al. (34) demonstrated that changes in scapular position can influence clearance in the subacro- mial space. Recent studies of subacromial clearance have not explicitly studied scapular motion but have either examined the effects of total humeral elevation in vivo $(2,9,33)$ or the effects of glenohumeral motion in a cadaver model $(3,8)$. Flatow et al. (8) reported that subacromial soft tissues were in contact with the undersurface of the acromion during normal elevation of the humerus and that suprahumeral space gradually decreased with simulated active elevation of the arm using cadaver specimens. This means that a decrease in scapular upward rotation with respect to the humerus served to decrease subacromial clearance. However, recent cadaver work in our laboratory has demonstrated that for a position of internal rotation at $90^{\circ}$ of elevation in the scapular plane, a decrease in upward rotation actually resulted in an increase in subacromial clearance. Therefore, it is unclear where the observed differences in scapular kinematics in the present study are detrimental (i.e., cause the pathology) or beneficial (i.e., help compensate for the pathology).

In the present study, the overall magnitude changes of scapular upward rotation in the subjects with impingement was between 2.5 and $4^{\circ}$ from 45 to $135^{\circ}$ of humeral elevation. Despite the small degree changes, these changes were not due to measurement error, rather, they represent true difference between the groups. The mean of the SEM value was $1.3^{\circ}$ from 45 to $135^{\circ}$ of humeral elevation, and the actual changes of the scapular upward rotation was almost 
two times beyond the SEM value (95\% of measurement error). Due to the possibility that the suprahumeral space might be less than $10 \mathrm{~mm}$, the effect of these small changes in scapular upward rotation might still create a crucial impact on the suprahumeral structures, especially due to the swimmers' requirement for repetitive overhead motions. In addition, the data collection process was carried out at the first half of the season where the training was not as intense and competitive. As the season progressed, muscles fatigue might become even higher. Consequently, the change in shoulder kinematics might be more drastic.

There are several limitations to the methods used in the present study. The advantages of using the Pro-360 inclinometer are its demonstrated reliability, validity, portability, and convenience. However, measurement of scapular kinematics in the present study was therefore only two dimensional, so the internal/external rotation and anterior/posterior tilting motions of the scapula were not measured, as was done in previous studies conducted in our lab $(19,35)$. Although three-dimensional studies could theoretically be carried out at pool side, at present, those techniques are better suited for a controlled laboratory environment. In the present study, the swimmers were only measured on $1 \mathrm{~d}$. It also would be interesting to see whether there are similar trends in other training days, especially during the second half of the season when the training is even more intense and competitive. However, Richardson et al. (30), utilizing a similar caliber of competitive swimmers, did find that $83 \%$ of the subjects in their study reported the greatest problem with shoulder pain during the early and middle portion of the season.

\section{REFERENCES}

1. Allegrucci, M., S. L. Whitney, and J. J. Irrgang. Clinical implications of secondary impingement of the shoulder in freestyle swimmers. J. Orthop. Sports Phys. Ther. 20:307-318, 1994.

2. Brossmann, J., K. W. Preidler, R. A. Pedowitz, L. M. White, D. TRudell, and D. Resnick. Shoulder impingement syndrome: influence of shoulder position on rotator cuff impingement: an anatomic study. AJR Am. J. Roentgenol. 167:1511-1515, 1996.

3. Burns, W. C. II, and T. L. Whipple. Anatomic relationships in the shoulder impingement syndrome. Clin. Orthop. 294:96-102, 1993.

4. Collins, H. R. Commentary on impingement syndrome in athletes. Am. J. Sports Med. 8:157-158, 1980.

5. CRotty, N. M., and J. SMith. Alterations in scapular position with fatigue: a study in swimmers. Clin. J. Sport Med. 10:251-258, 2000.

6. Doody, S. G., L. Freedman, and J. C. Waterland. Shoulder movements during abduction in the scapular plane. Arch. Phys. Med. Rehabil. 51:595-604, 1970.

7. Endo, K., T. Ikata, S. Катон, and Y. TakedA. Radiographic assessment of scapular rotational tilt in chronic shoulder impingement syndrome. J. Orthop. Sci. 6:3-10, 2001.

8. Flatow, E. L., L. J. Soslowsky, J. B. Ticker, et al. Excursion of the rotator cuff under the acromion: patterns of subacromial contact. Am. J. Sports Med. 22:779-788, 1994.

9. Graichen, H., H. Bonel, T. Stammberger, K. H. Englmeier, M. REISER, and F. ECKSTEIN. Subacromial space width changes during abduction and rotation: a 3-D MR imaging study. Surg. Radiol. Anat. 21:59-64, 1999.
Additionally, there are also limitations with regards to the subject population. Due to the fact that subjects were evaluated at poolside, our diagnosis of impingement was made base solely on physical exam. Although we attempted to selectively recruited swimmers without shoulder instability, it is possible that some subjects may have had subtle instability (so-called micro instability) that is commonly found in swimmers $(20,38)$. Additionally, AC joint pathology and scapular winging were not ruled out. Therefore the result of this study may not be applicable to all swimmers with shoulder impingement syndrome. Finally, the swimmers who participated in this study were between 18 and 35; therefore, the result may not be generalized to other populations of swimmers.

\section{CONCLUSIONS}

The preswim measurements of scapular upward rotation in the two groups were similar. However, in the subjects with impingement, the amount of scapular upward rotation at 45,90 , and $135^{\circ}$ of humeral elevation decreased after swim training. The greatest decrease in scapular upward rotation was at $135^{\circ}$. These findings suggest that the scapular kinematics of swimmers with shoulder impingement syndrome may not have changed until after they practiced swimming and fatigued the shoulder muscles. The clinical significance of these differences is still unknown.

We would like to thank all of the swim coaches that helped with subject recruitment (especially Bob Piotti, Dick Jackson, and Bruce Bronsdon), the Garden State Master Swimmers, and the Delaware Valley Master Swimmers newsletter editors who offered their enthusiasm in recruiting subjects.

10. Graichen, H., T. Stammberger, H. Bonel, et al. Three-dimensional analysis of shoulder girdle and supraspinatus motion patterns in patients with impingement syndrome. J. Orthop. Res. 19:1192-1198, 2001.

11. Greenfield, B., P. A. Catlin, P. W. Coats, E. Green, J. J. McDonald, and C. NoRth. Posture in patients with shoulder overuse injuries and healthy individuals. J. Orthop. Sports Phys. Ther. 21:287-295, 1995.

12. Hebert, L. J., H. Moffet, B. J. McFadyen, and C. E. Dionne. Scapular behavior in shoulder impingement syndrome. Arch. Phys. Med. Rehabil. 83:60-69, 2002.

13. Johnson, J. E., F. H. Sim, and S. G. ScotT. Musculoskeletal injuries in competitive swimmers. Mayo Clin. Proc. 62:289-304, 1987.

14. Johnson, M. P., P. W. McCluRe, and A. R. Karduna. New method to assess scapular upward rotation in subjects with shoulder pathology. J. Orthop. Sports Phys. Ther. 31:81-89, 2001.

15. Karduna, A. R., P. J. Kerner, and M. D. Lazarus. Contact forces in the subacromial space: effects of scapular orientation. J. Shoulder Elbow Surg. (in press).

16. Kennedy, J. C., R. Hawkins, and W. B. Krissoff. Orthopaedic manifestations of swimming. Am. J. Sports Med. 6:309-322, 1978.

17. Ludewig, P. M., and T. M. CooK. Alterations in shoulder kinematics and associated muscle activity in people with symptoms of shoulder impingement. Phys. Ther. 80:276-291, 2000.

18. Lukasiewicz, A. C., P. McClure, L. Michener, N. Pratt, and B. SenNeTt. Comparison of 3-dimensional scapular position and orientation between subjects with and without shoulder impingement. J. Orthop. Sports Phys. Ther. 29:574-583, 1999.

19. McClure, P. W., L. A. Michener, B. J. Sennett, and A. R. KARDUNA. Direct 3-dimensional measurement of scapular kine- 
matics during dynamic movements in vivo. J. Shoulder Elbow Surg. 10:269-277, 2001.

20. McMaster, W. C., A. Roberts, and T. Stoddard. A correlation between shoulder laxity and interfering pain in competitive swimmers. Am. J. Sports Med. 26:83-86, 1998.

21. McMaster, W. C., and J. Troup. A survey of interfering shoulder pain in United States competitive swimmers. Am. J. Sports Med. 21:67-70, 1993 .

22. NeER, C. S. II. Anterior acromioplasty for the chronic impingement syndrome in the shoulder. J. Bone Joint Surg. Am. 54A:41-50, 1972.

23. NeER, C. S. II, and R. P. Welsh. The shoulder in sports. Orthop. Clin. North Am. 8:583-591, 1977.

24. Neumann, D. A. Kinesiology of the Musculoskeletal System. St. Louis: Mosby, 2002, pp. 122-124.

25. Norkin, C. C., and P. K. Levangie. Joint Structure and Function: A Comprehensive Analysis. Philadelphia, F. A. Davis Co., 1992, pp. 230-232.

26. Perry, J., M. Pink, F. W. Jobe, J. Kerrigan, A. Browne, and M. L. Scovazzo. The painful shoulder during the backstroke: an EMG and cinematographic analysis of 12 muscles. Clin. J. Sport Med. 2:13-20, 1992.

27. Pink, M., F. W. Jobe, J. Perry, A. Browne, M. L. Scovazzo, and J. KerRigan. The painful shoulder during the butterfly stroke: an electromyographic and cinematographic analysis of twelve muscles. Clin. Orthop. 288:60-72, 1993.

28. Pink, M., J. Perry, A. Browne, M. L. Scovazzo, and J. Kerrigan. The normal shoulder during freestyle swimming: an electromyographic and cinematographic analysis of twelve muscles. Am. J. Sports Med. 19:569-576, 1991.
29. Portney, L. G., and M. P. Watkins. Foundations of Clinical Research: Application to Practice, 2nd Ed. Upper Saddle River, NJ: Prentice Hall Health, 2000, p. 565.

30. Richardson, A. B., F. W. Jobe, and H. R. Collins. The shoulder in competitive swimming. Am. J. Sports Med. 8:159-163, 1980.

31. Rupp, S., K. Berninger, and T. Hopf. Shoulder problems in high level swimmers: impingement, anterior instability, muscular imbalance? Int. J. Sports Med. 16:557-562, 1995.

32. Scovazzo, M. L., A. Browne, M. Pink, F. W. Jobe, and J. KerRIGAN. The painful shoulder during freestyle swimming: an electromyographic cinematographic analysis of twelve muscles. Am. J. Sports Med. 19:577-582, 1991.

33. Shibuta, H., K. TAmai, and K.-I. TABuchi. Magnetic resonance imaging of the shoulder in abduction. Clin. Orthop. 348:107-113, 1998.

34. Solem-Bertoft, E., K.-Ä. Thuomas, and C.-E. Westerberg. The influence of scapula retraction and protraction on the width of the subacromial space: an MRI study. Clin. Orthop. 296:99-103, 1993.

35. Tsai, N. T., P. W. McClure, and A. R. Karduna. Effects of muscle fatigue on 3-dimensional scapular kinematics. Arch. Phys. Med. Rehabil. 84:1000-1005, 2003.

36. Yanai, T., and J. G. Hay. Shoulder impingement in front-crawl swimming: II. Analysis of stroking technique. Med. Sci. Sports Exerc. 32:30-40, 2000.

37. Youdas, J. W., J. R. Carey, T. R. Garrett, and V. J. Suman. Reliability of goniometric measurements of active arm elevation in the scapula plane obtained in a clinical setting. Arch. Phys. Med. Rehabil. 75:1137-1144, 1994.

38. ZemeK, M. J., and D. J. Magee. Comparison of glenohumeral joint laxity in elite and recreational swimmers. Clin. J. Sport Med. 6:40-47, 1996. 